\title{
Electrostatic Potential Measurement by Using 6-MeV Heavy Ion Beam Probe on LHD*)
}

\author{
Takeshi IDO, Akihiro SHIMIZU, Masaki NISHIURA, Shinji KATO, Haruhisa NAKANO, \\ Akimitsu NISHIZAWA $^{1)}$, Yasuji HAMADA, Mitsuhiro YOKOTA, Kiwamu TSUKADA, \\ Hideki OGAWA, Tomoyuki INOUE, Katsumi IDA, Mikiro YOSHINUMA, \\ Sadayoshi MURAKAMI ${ }^{2)}$, Kenji TANAKA, Kazumichi NARIHARA, Ichihiro YAMADA, \\ Kazuo KAWAHATA, Naoki TAMURA and the LHD Experimental Group \\ National Institute for Fusion Science, 322-6, Oroshi, Toki, Gifu, 509-5292, Japan \\ ${ }^{1)}$ Tono Geoscience Centre, Japan Atomic Energy Agency, Toki, Gifu, Japan. \\ ${ }^{2)}$ Department of Nuclear Engineering, Kyoto University, Kyoto 606-8501, Japan
}

(Received 7 December 2007 / Accepted 8 April 2008)

\begin{abstract}
A heavy ion beam probe (HIBP) using a $3 \mathrm{MV}$ tandem accelerator was installed in Large Helical Device (LHD). It is designed to measure the electrostatic potential in the core region directly. It is calibrated and can be used to measure the electrostatic potential profiles in LHD plasmas. The radial electric field $\left(E_{\mathrm{r}}\right)$ obtained from the potential profiles measured using the HIBP agrees with that measured by charge exchange spectroscopy (CXS). $E_{\mathrm{r}}$ predicted by the neoclassical theory is also compared to that measured using the HIBP, and is in good agreement with the experimental results in the core region.
\end{abstract}

(c) 2008 The Japan Society of Plasma Science and Nuclear Fusion Research

Keywords: heavy ion beam probe, HIBP, electrostatic potential, electric field

DOI: $10.1585 /$ pfr.3.031

\section{Introduction}

A heavy ion beam probe (HIBP) is a unique diagnostic tool for measuring the electrostatic potential, its fluctuations and density fluctuations in high-temperature magnetically confined plasmas directly without perturbing the plasmas. HIBPs have been installed in various types of devices [1-16] and useful results have been obtained.

In non-axisymmetric plasmas, the radial electric field $\left(E_{\mathrm{r}}\right)$ plays crucial roles in transport phenomena. For example, the electron confinement is improved in the core region during electron cyclotron heating (ECH) [17-22]. This is considered to be related to the transition from the ion-root to the electron-root of $E_{\mathrm{r}}$ predicted by the neoclassical theory, and called the Core Electron Root Confinement (CERC) [23]. Recently, meso-scaled structure of $E_{\mathrm{r}}$, termed as zonal flow, is confirmed to exist in plasmas universally, and shown to affect the radial transport of plasmas by regulating turbulence. Therefore, understanding the behavior of $E_{\mathrm{r}}$ and its effects on transport is important for predicting the performance of future fusion plasmas. To achieve this, an HIBP has been developed for Large Helical Device (LHD) [24-26].

In this article, the apparatus of the HIBP in LHD (referred to as LHD-HIBP) is described in Sec. 2. The measured potential profiles are shown in Sec. 3. $E_{\mathrm{r}}$ measured

author'se-mail:ido@LHD.nifs.ac.jp

*) This article is based on the invited talk at the 24th JSPF Annual Meeting (2007, Himeji). using the HIBP is compared with that estimated by charge exchange spectroscopy (CXS), which is another reliable tool for determining $E_{\mathrm{r}}$ by measuring the velocity of the plasma flow. $E_{\mathrm{r}}$ calculated based on the neoclassical theory is also compared with the results obtained using the HIBP. In Sec. 4, the future prospects of fluctuation measurement are discussed. The results are summarized in Sec. 5 .

\section{LHD-HIBP System}

\subsection{System overview}

In an HIBP, singly charged ions are injected into a plasma as a primary beam, and doubly charged ions obtained by ionization due to collision with the plasma on its path are detected (referred to as the secondary beam). Since the energy difference between the primary and secondary beams is equivalent to the electrostatic potential energy at the position where the secondary beam is produced, we can obtain the plasma potential locally by measuring the secondary beam energy.

In order to extract the probing beam from the plasma, the Larmor radius of the probing beam of the HIBP must be comparable with the minor radius of the plasma device. The necessary beam energy $\left(E_{\mathrm{b}}\right)$ is scaled approximately as $E_{\mathrm{b}} \propto a^{2} B^{2} / m_{\mathrm{b}}$, where $a, B$, and $m_{\mathrm{b}}$ are the size of the plasma device, magnetic field strength, and mass of the beam ion, respectively. Since the magnetic field in LHD is approximately up to $3 \mathrm{~T}$ and a Larmor radius of a few meters is necessary, singly charged gold ions $\left(\mathrm{Au}^{+}\right)$ 


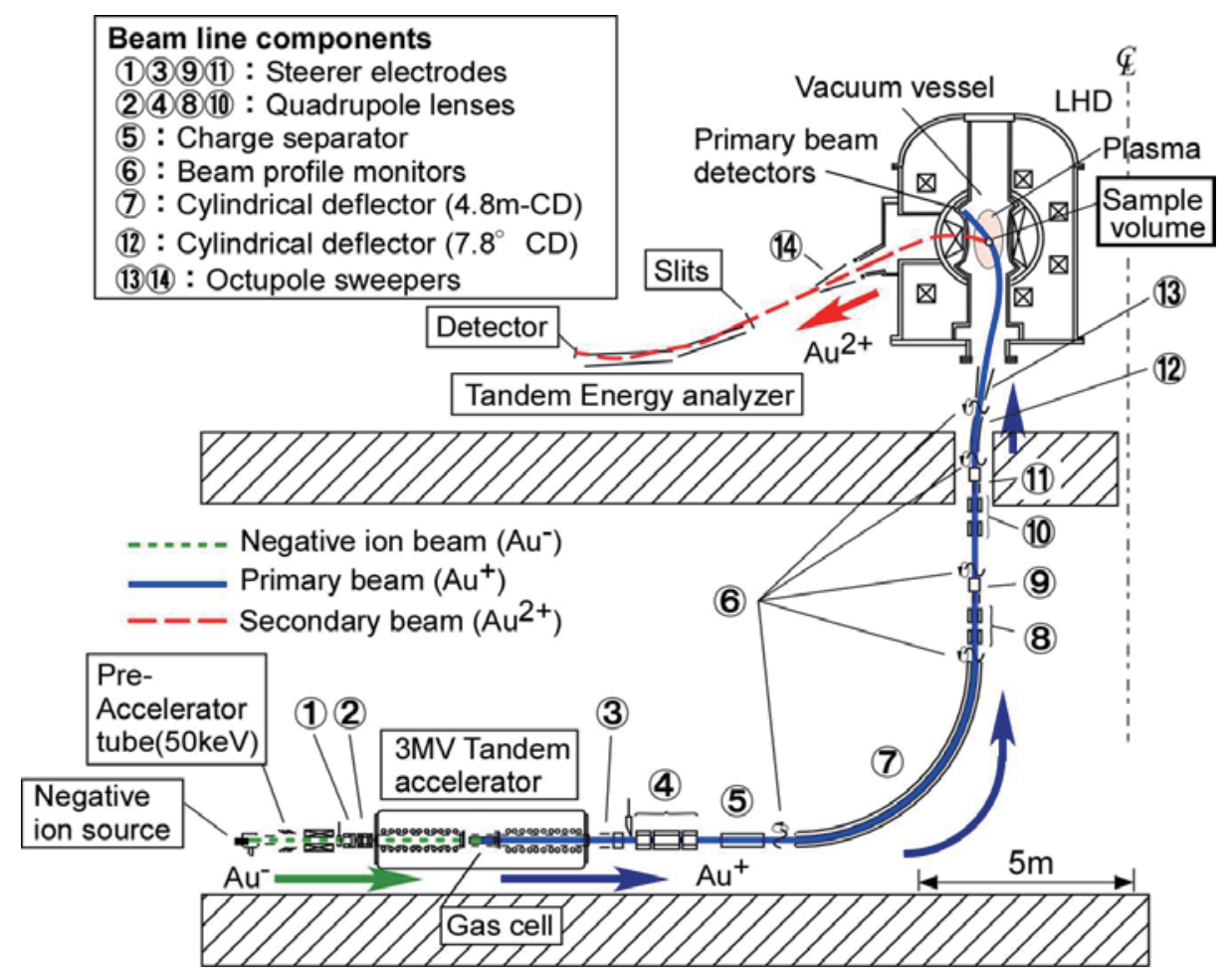

Fig. 1 Schematic of the LHD-HIBP system.

with energies of approximately up to $6 \mathrm{MeV}$ are required as the primary beam. Thus far, the 2-MeV HIBP in TEXT$\mathrm{U}$ was the highest energy HIBP available, and there were some technical difficulties relating to high-voltage operation. Thus, in order to avoid difficulties, a tandem accelerator and a new type of energy analyzer have been adopted in the LHD-HIBP. They are described in following subsection.

Figure 1 shows a schematic of the LHD-HIBP system. The primary beam $\left(\mathrm{Au}^{+}\right)$is injected from a lower vertical port of LHD, and the secondary beam ionized in the vacuum vessel $\left(\mathrm{Au}^{2+}\right)$ is extracted from a horizontal port and its energy is analyzed. The injection and detection positions are designed so as to measure the potential at the magnetic axis in the standard magnetic configuration of LHD, in which the major radius of the magnetic axis $\left(R_{\mathrm{ax}}\right)$ is $3.75 \mathrm{~m}$, quadrupole magnetic field component $\left(B_{\mathrm{q}}\right)$ is $100 \%$, and pitch parameter of the helical coil $(\gamma)$ is 1.254 .

The observation position can be selected by changing the combination of magnetic field strength, beam energy, and injection angles. Observable positions are shown in Fig. 2. Actual observation positions are distributed in the toroidal direction and the positions in the figure are projected on a poloidal cross section by tracing the magnetic field line from the actual observation point. For example, when the toroidal magnetic field strength $\left(B_{\mathrm{t}}\right)$ is $3.0 \mathrm{~T}$ and $R_{\mathrm{ax}}$ is $3.75 \mathrm{~m}$, the potential profile on the curve passing through the magnetic axis can be measured by sweeping
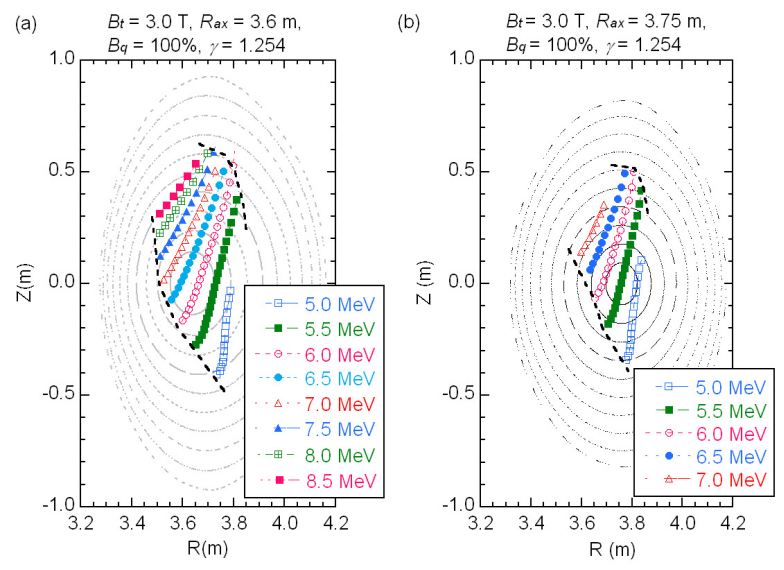

Fig. 2 Observable region. The sample volume traces the marked curve during a sweep of the probing beam. The sample volumes of the probing beam with the same Larmor radius $\left(L_{\mathrm{b}} \propto \sqrt{E_{\mathrm{b}}} / B_{\mathrm{t}}\right)$ trace the same curve, where $E_{\mathrm{b}}$ and $B_{\mathrm{t}}$ are the beam energy and the toroidal magnetic field strength, respectively. (a) $R_{\mathrm{ax}}=3.6 \mathrm{~m}, B_{\mathrm{q}}=100 \%$, $\gamma=1.254$. (b) $R_{\mathrm{ax}}=3.75 \mathrm{~m}, B_{\mathrm{q}}=100 \%, \gamma=1.254$.

a probing beam with an energy of $5.5 \mathrm{MeV}$. The lower and upper sides of the observable region are limited by the injection and extraction ports, respectively; thus the LHDHIBP can measure the potential in the core region of plas- 
mas.

Measurement with high temporal resolution is one of the advantages of HIBPs. Currently, the temporal resolution of the LHD-HIBP is about $100 \mu \mathrm{s}$, which is limited by the frequency response of the high-gain amplifiers $(-3 \mathrm{~dB}$ at $15 \mathrm{kHz}$ ). Although the frequency response is not sufficient to measure micro-turbulence, fast transitions due to electric bifurcation and low-frequency MHD modes can be detected marginally.

In the following subsections, some components of the LHD-HIBP are described.

\subsection{Ion source and $3 \mathrm{MV}$ tandem accelerator}

A MeV-range beam is required for the LHD-HIBP as described above. To reduce the acceleration voltage, a tandem accelerator is employed to obtain this high-energy beam, unlike previous HIBPs in which single-ended accelerators have been used. The tandem accelerator includes an ionizer, which is a gas cell for the LHD-HIBP, at the center, and high positive voltage is applied there. Singly charged negative ions are initially injected into the accelerator and accelerated toward the gas cell. They are ionized to positive ions by collisions with neutral particles in the gas cell. Then, they are accelerated again toward a port on the other side, which is grounded. Thus, the tandem accelerator can accelerate the beam particles twice, and the required acceleration voltage can be reduced to half of that corresponding to the required beam energy. In the LHD-HIBP, the acceleration voltage is up to $3 \mathrm{MV}$ and singly charged positive ions with energies of up to $6 \mathrm{MeV}$ can be extracted. The stability of the acceleration voltage, expressed in terms of root-mean-square deviations (RMSD), is $5 \mathrm{~V}$ for an applied voltage of $0.779 \mathrm{MV}$ in a magnetic field of $1.5 \mathrm{~T}$, and $250 \mathrm{~V}$ for an applied voltage of $2.635 \mathrm{MV}$ in a magnetic field of $2.829 \mathrm{~T}$. Since the fluctuations of the energy of the probing beam are double the above values, they should be considered in potential measurement, especially in strong magnetic fields $(>2.0 \mathrm{~T})$.

A tandem accelerator requires negative ions. Hence, plasma-sputter-type negative ion sources have been developed since the development of the LHD-HIBP [27-29]. At present, the output current is about $12 \mu \mathrm{A}$ under the steady operation. Since the conversion efficiency from $\mathrm{Au}^{-}$to $\mathrm{Au}^{+}$in the gas cell of the accelerator is $\leq 20 \%$, the current of the primary beam is a few microamperes.

The energy spread of the negative ion beam from the ion source is measured as $8 \mathrm{eV}$ [28]. Since the negative ions are ionized in the gas cell installed in the tandem accelerator, the collisions with the neutral gas also cause energy spread of the beam. The energy spread in the gas cell is estimated to be $\leq 20 \mathrm{eV}$ or less [30]. It will not affect potential measurement because the change in the average beam energy is measured for the HIBP, and the number of the detected particles is sufficient for measurement of the average energy $\left(\geq 1000 \mu \mathrm{s}^{-1}\right)$.

\subsection{Beam transport system}

The accelerator is installed in the basement of the LHD building for convenient maintenance and to minimize the influence of the stray magnetic field from LHD. However, this increases the length of the beam transport line for beam injection becomes long (about $20 \mathrm{~m}$ ).

Because the stray magnetic field deflects the beam trajectory by several centimeters in the beam line, steering electrodes are installed to compensate the deflection at Nos. 9 and 11 in Fig. 1.

Adjustment of beam focusing is essential for optimizing the size of sample volumes. Thus, electrostatic quadrupole lenses are installed (No.10 in Fig. 1). They are arranged as a focusing-defocusing pair; hence, the focus of the beam can be adjusted in two dimensions independently. The beam profiles are measured with two-dimensional rotating wires shown as No.6 in Fig. 1. The beam divergence can be estimated by changing the optical characteristics of the lenses and measuring the beam profile. In the experiments described in this paper, a beam with a diameter of about $10 \mathrm{~mm}$ at the injection port is adjusted to be focused at the detector for the secondary beam, and the size of the sample volume is about $<40 \mathrm{~mm}$ in the minor radius direction [31], which determines the spatial resolution.

\subsection{Octupole sweepers}

The position of the sample volume can be selected by changing the incident angle to plasmas. Unlike trajectories of the probing beam in axisymmetric devices, which are mostly on a poloidal plane, the probing beams in helical systems move toward the toroidal direction as well as on a poloidal plane. Thus, the incident angle must be controlled in two dimensions. In addition, the incident angle of the secondary beam to the energy analyzer can change during a sweep of the probing beam, which possibly causes errors in the analysis of beam energy. Therefore, in the LHD-HIBP, two octupole sweepers are installed at the injection and extraction ports. The sweeper at the injection and extraction ports serve to adjust the incident angles to the plasma and the energy analyzer, respectively, and latter one is to adjust the incident angles to the energy analyzer. This method has been developed in an HIBP in the Compact Helical System (CHS) [32]. The sweep frequency of the applied voltage is $10 \mathrm{~Hz}$ which is limited by the maximum current of the high-voltage amplifiers. Thus, the potential profile can be measured with a repetition frequency of $20 \mathrm{~Hz}$.

The octupole electrodes are horn shaped to deflect the beam most effectively. As a result, the electric field among the electrodes has a three-dimensional structure. Since the measurement positions of the HIBP are usually estimated only by trajectory calculations, the threedimensional structure of the electric fields should be considered in the trajectory calculation. In order to simulate the beam trajectory precisely, the electric field is calculated 
by the three-dimensional boundary element method. The results of the unit-tests of the octupole sweeper confirm that the calculation can simulate the electric field correctly. The calibration of the trajectory of the probing beam in LHD has been described in Ref. [33]. The results indicate that the probing beam can be detected using the calculated sweep voltages, although small deviations due to the stray magnetic field should be compensated.

\subsection{Tandem energy analyzer}

The change in the electrostatic potential in plasmas is obtained from analysis of the change in energy of the secondary beam. In traditional HIBPs, parallel plate electrostatic analyzers [34] have been used because they have the desirable property of second-order focusing for the incident angle. However, this requires an impractical voltage of several hundred $\mathrm{kV}$ for the MeV-range beam of the LHD-HIBP. Hence, we have devised a new energy analyzer with tandem electrodes $[25,35]$ to reduce applied voltages and to maintain the second-order focusing for the incident angle available in the LHD-HIBP. The designed incident angle of the traditional parallel plate analyzer is 30 degree, but the incident angles of the new tandem energy analyzer are designed as 6 degrees for the first electrode and 10 degrees for the second electrode. Consequently, the required voltages can be reduced to $56.5 \mathrm{kV}$ on the first electrode and $113.6 \mathrm{kV}$ on the second electrode for a 6 $\mathrm{MeV}$ beam.

The energy change $\left(\Delta E_{\mathrm{b}}\right)$ is analyzed on the basis of the displacement of the beam on the detector as $\Delta E_{\mathrm{b}}=$ $-2.27 \times 10^{-3} \cdot E_{\mathrm{b}} \cdot H_{\text {slit }} \cdot \Delta N D$, where $E_{\mathrm{b}}$ is the energy of the primary beam, $H_{\text {slit }}$ is the height of the opening of the entrance slit, and $N D$ is the displacement normalized by the height of the slit [25]. The height of the slit opening is set to $3 \mathrm{~mm}$ in view of the intensity of the detected beam and the spatial resolution. Thus, the dynamic range of the potential measurement is $6.81 \times 10^{-3} \cdot E_{\mathrm{b}}$.

Dependence of energy analysis on the incident angle is undesirable for HIBPs because the incident angle can change while the probing beam is swept to measure the potential profile, which can cause errors in energy analysis. The incident angle dependence of energy analysis is shown in Fig. 3. The vertical axis is the displacement of the beam on the detector, which is normalized by the height of the slit opening, and the beam energy is estimated from the normalized displacement. Since the geometry of the electrodes in the beam line limits the incident angle to 0.5 degrees, the incident-angle dependence is acceptable for potential measurement. The beam energy is analyzed with an accuracy of $<63 \mathrm{eV}$ for a $1.5-\mathrm{MeV}$ beam even if the incident angle fully changes.

\subsection{Calibration for potential measurement}

The magnetic field in LHD is also essential for the HIBP operation. Therefore, calibration of the integrated

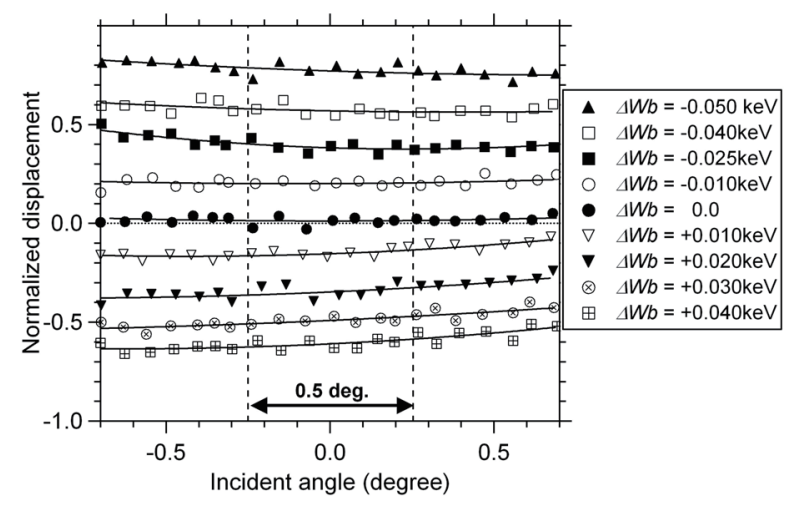

Fig. 3 Results of calibration of the energy analyzer using a 30$\mathrm{keV}$ thallium beam. The horizontal axis is the change in the incident angle from the designed incident angle $(6 \mathrm{de}-$ grees). The vertical axis is the displacement of the beam on the detector and is normalized by the height of the opening of the entrance slit. The slit height is $1 \mathrm{~mm}$ in this calibration. The change in the beam energy is estimated from the change in the normalized position. Each marked line shows the incident angle dependence of the energy analysis. The beam energy is shown in the legend as the deviation from $30 \mathrm{keV}$.

HIBP system, which consists of the ion source, the accelerator, the beam line components, the magnetic field in LHD, and the energy analyzer, is necessary for the potential measurement. In calibration experiments, the secondary beam ionized by collisions with the neutral gas injected into the vacuum vessel is used. Since there is no electric field in the vacuum vessel when the plasma is not produced, the secondary beam energy is identical to the incident energy of the primary beam. Thus, it can be controlled by adjusting the primary beam energy. In LHD, a small amount of neutral gas injected from the NBI port is available for calibration in every discharge.

Figure 4 shows the analyzed secondary beams energy when the primary beam energy is varied from $4.258 \mathrm{MeV}$ $(-2.0 \mathrm{keV})$ to $4.262 \mathrm{MeV}(+2.0 \mathrm{keV})$. In the experiment, hydrogen gas is used as the fuel gas and the gas pressure is in the range of $10^{-3}$ to $10^{-2} \mathrm{~Pa}$ in the vacuum vessel of LHD. Figure 4 indicates that the change in beam energy is analyzed successfully, and the results verify the linearity of the energy analysis.

In plasma experiments, a probing beam is deflected by a poloidal magnetic field due to plasma current. The deflection can cause two types of errors in potential measurement using an electrostatic energy analyzer. One is based on the principle of the energy analyzer. Since the beam energy is analyzed using the flight distance of the beam in the energy analyzer, only the velocity component in the plane containing the designed beam trajectory in the analyzer can be analyzed. The plane is vertical in the LHDHIBP. Thus, if the beam is deflected in the horizontal (toroidal) direction by the poloidal magnetic field, a de- 


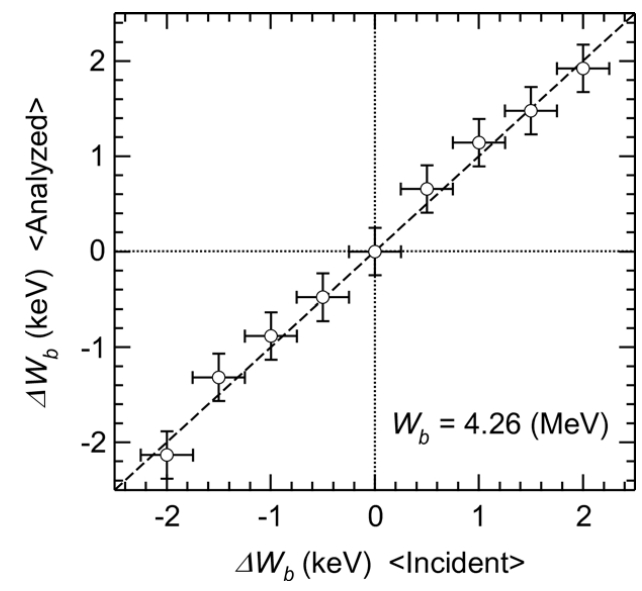

Fig. 4 Comparison between the incident and analyzed beam energies. The horizontal and vertical axes are the incident and analyzed beam energies, respectively. The energies are expressed as the difference from $4.262 \mathrm{MeV}$.

crease in the analyzed beam energy is observed, which corresponds to the horizontal velocity. In the LHD-HIBP, the distance between the center of the detector and the plasma edge is $11 \mathrm{~m}$ and the width of the detector is $80 \mathrm{~mm}$ in the horizontal direction, thus the horizontal angle of the detectable beam $(\alpha)$ is limited mechanically to $7.3 \times 10^{-3}$ $\mathrm{rad}$, even if the beam is assumed to have no width and divergence. The maximum error caused by the deflection is $\Delta E_{\mathrm{b}} / E_{\mathrm{b}}=1-\cos ^{2} \alpha=5.3 \times 10^{-5}$, which corresponds to $80-\mathrm{eV}$ change in the probing beam when the incident energy is $1.5 \mathrm{MeV}$. The error is estimated under extreme conditions; the horizontal movement of the beam on the detector is less than $1 / 50$ of the above assumption. Therefore, the deflection due to the poloidal magnetic field does not affect potential measurement.

The other error caused by deflection is due to the inadequate alignment of the analyzer. In the analyzer, the beam is selected by rectangular slits. The dimensions of the slit opening are $3 \mathrm{~mm}$ in height (the electric field direction in the analyzer) and $100 \mathrm{~mm}$ in width (horizontal direction). The width of the slit is wider than that of the secondary beam, so that the beam can be detected even if it is deflected by the poloidal magnetic field. In principle, the horizontal displacement of the beam should never affect energy analysis. However, if the axes of the slit and detector are not aligned with adequate accuracy, horizontal movement causes an error in energy analysis. Hence, we examined its influence by the sweeping the beam horizontally with the octupole sweeper shown as No.14 in Fig. 1. Figure 5 (a) shows the displacement of the beam on the detector during a horizontal sweep, and indicates that the displacement in the horizontal deflection is linear with the change in the sweep voltage. The displacement in the vertical direction, with which the energy change is analyzed, is small but finite. This suggests that the deflection in (a)

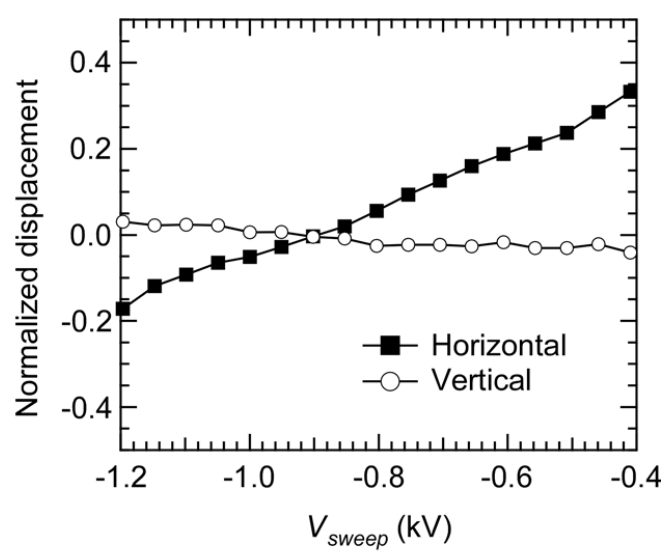

(b)

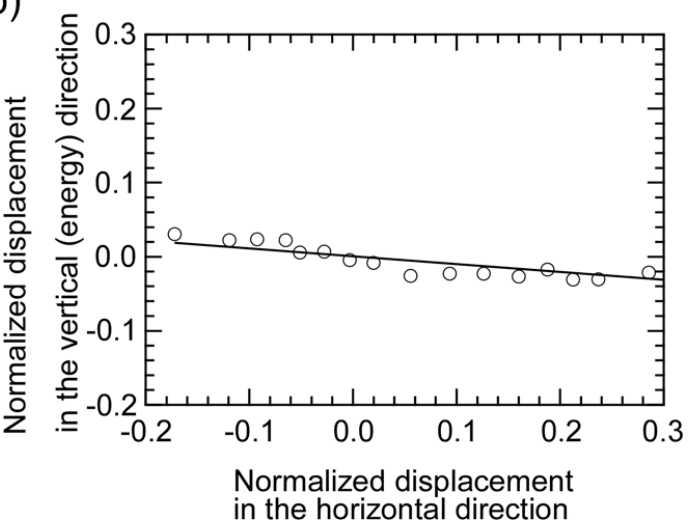

Fig. 5 (a) Normalized displacement of the beam during a horizontal sweep. (b) Relationship between horizontal and vertical displacements.

the horizontal direction causes an error in energy analysis. The vertical displacement $(V D)$ is related to the horizontal $(H D)$ as $V D=-0.10 H D$ (Fig. 5 (b)). Therefore, this error must be considered in potential measurement.

\section{Potential Profile Measurement 3.1 Experimental condition}

Experiments for comparison between the HIBP and CXS were performed in a magnetic configuration where $B_{\mathrm{t}}=1.5 \mathrm{~T}, R_{\mathrm{ax}}=3.6 \mathrm{~m}, B_{\mathrm{q}}=100 \%$, and $\gamma=1.245$. Hydrogen is used as the fuel gas. The energy of the probing beam of the HIBP is $1.562 \mathrm{MeV}$, and the sample volume traces on the curve shown in Fig. 6(a) during a sweep of the beam. Figure 6 (b) shows the timing of NBI and ECH, and temporal evolution of the line averaged electron density. The plasma is produced and sustained by tangential NBI of 3.8 MW, and ECH of 1.1 MW is superposed from 1.5 to $2.1 \mathrm{~s}$. Perpendicular NBI of $1.2 \mathrm{MW}$ is modulated to measure the profile of the poloidal flow by CXS.

The probing beam of the HIBP is swept at a frequency of $10 \mathrm{~Hz}$. The secondary beam current is shown in Fig. 6(c). The secondary beam is detected before the plasma production $(\mathrm{t}<0.7 \mathrm{~s})$, and data for calibration using the secondary beam is obtained. The temperature and density profiles are shown in Fig. 6(d) and (e). 

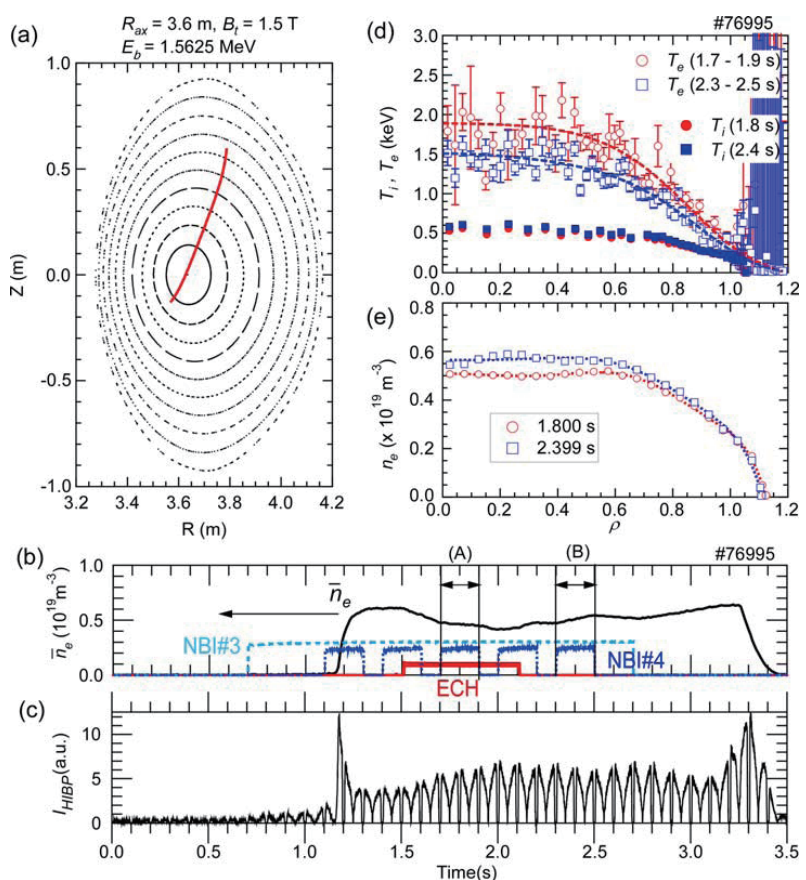

Fig. 6 (a) Observation position (red curve) and the magnetic flux surface (black dots). The sample volume traces the red curve during a sweep of the probing beam. (b) Timing of $\mathrm{NBI}$ and $\mathrm{ECH}$, and temporal evolution of the line averaged electron density. (A) and (B) indicate periods for which the potential and $E_{\mathrm{r}}$ profiles are shown. (c) The secondary beam current. The beam is swept at a frequency of $10 \mathrm{~Hz}$. (d) Electron and ion temperature profiles measured by Thomson scattering and CXS. (e) Density profiles reconstructed from line averaged density measured using an FIR interferometer.

\subsection{Error estimation}

First, we estimate the errors in potential measurement. Possible error sources are fluctuations of the acceleration and analyzer voltages, and electric noise of the amplifier.

The error due to electric noise of the amplifier depends on the intensity of the detected beam. The ratio of the noise to the beam intensity is about 0.025 in the core region of the plasma, which corresponds to an error of about $340 \mathrm{~V}$ in potential measurement.

The fluctuation in the acceleration voltage is shown in Fig. 7 (a). The maximum fluctuation in the acceleration voltage is $80 \mathrm{eV}$, which is caused by a voltage-drop in the commercial power source due to the operation of the heating apparatuses. The influence of this fluctuation can be removed from the detected signal.

The fluctuation in the analyzer voltages is shown in Fig. 7 (b), and is less than the noise level (2.5 V RMSD), which corresponds to $0.25 \mathrm{kV}$ RMSD change in beam energy. It is less than the noise level of the amplifiers.

The toroidal deflection due to the change in the poloidal magnetic field is shown in Fig. 7 (c). The maximum of error in potential measurement is $50 \mathrm{eV}$, and the error can be corrected in the potential profiles. The loss of beam energy through collisions with plasma particles
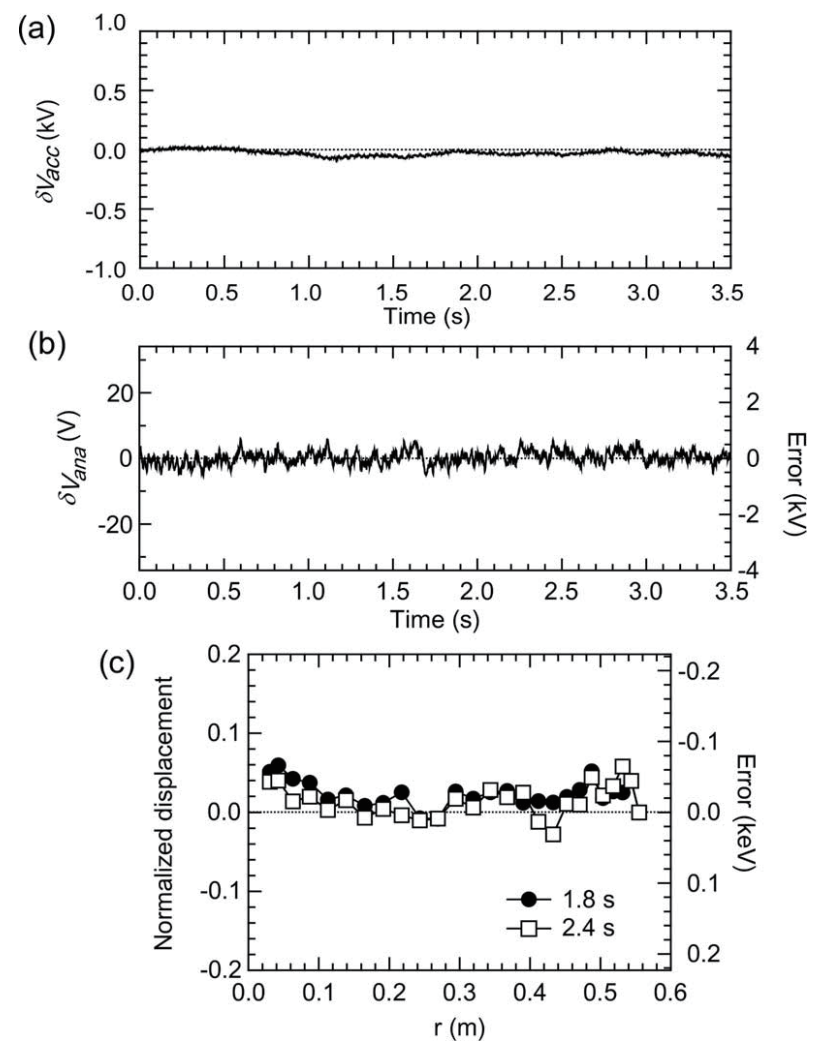

Fig. 7 Error analysis of the HIBP signal. (a) Stability of the acceleration voltage. (b) Stability of the analyzer voltage. (c) Effect of the toroidal displacement. The horizontal axis is the position of the sample volume which is projected on a horizontally elongated poloidal cross-section by tracing the magnetic field line from the actual observation point.

might cause an error, but it is estimated to be $<10 \mathrm{eV}$ [24] because the plasma density is low.

In previous HIBPs, the radiation from plasmas caused errors occasionally because it produced photoelectrons in the beam line, which caused drops in the sweep and analyzer voltages and pseudo signals in the detectors. In the experiments described in this paper, however, no signal due to the radiation is observed while the probing beam is not injected. Thus, the radiation does not affect the signal.

Therefore, the error in potential measurement is estimated to about $340 \mathrm{~V}$.

\subsection{Comparison among HIBP, CXS, and neoclassical calculation}

The potential profiles measured using the HIBP are shown in Fig. 8 (a). During superposition of ECH (1.7 s), the potential varies in the positive direction. The gradient of the potential increases especially in the region of $\rho>$ $0.4 \mathrm{~m}$, which indicates that a positive $E_{\mathrm{r}}$ is formed there.

In Fig. 8 (b), $E_{\mathrm{r}}$ obtained from the derivative of the potential profile measured using the HIBP is compared with that estimated from the poloidal flow measured by CXS. Although $E_{\mathrm{r}}$ is not obtained by CXS for $\rho<0.6$ because CXS depends on the emission profile and the emission 
(a)

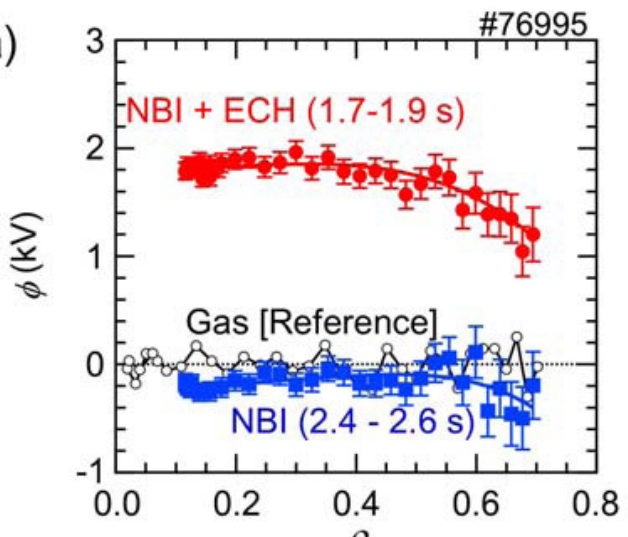

(b)
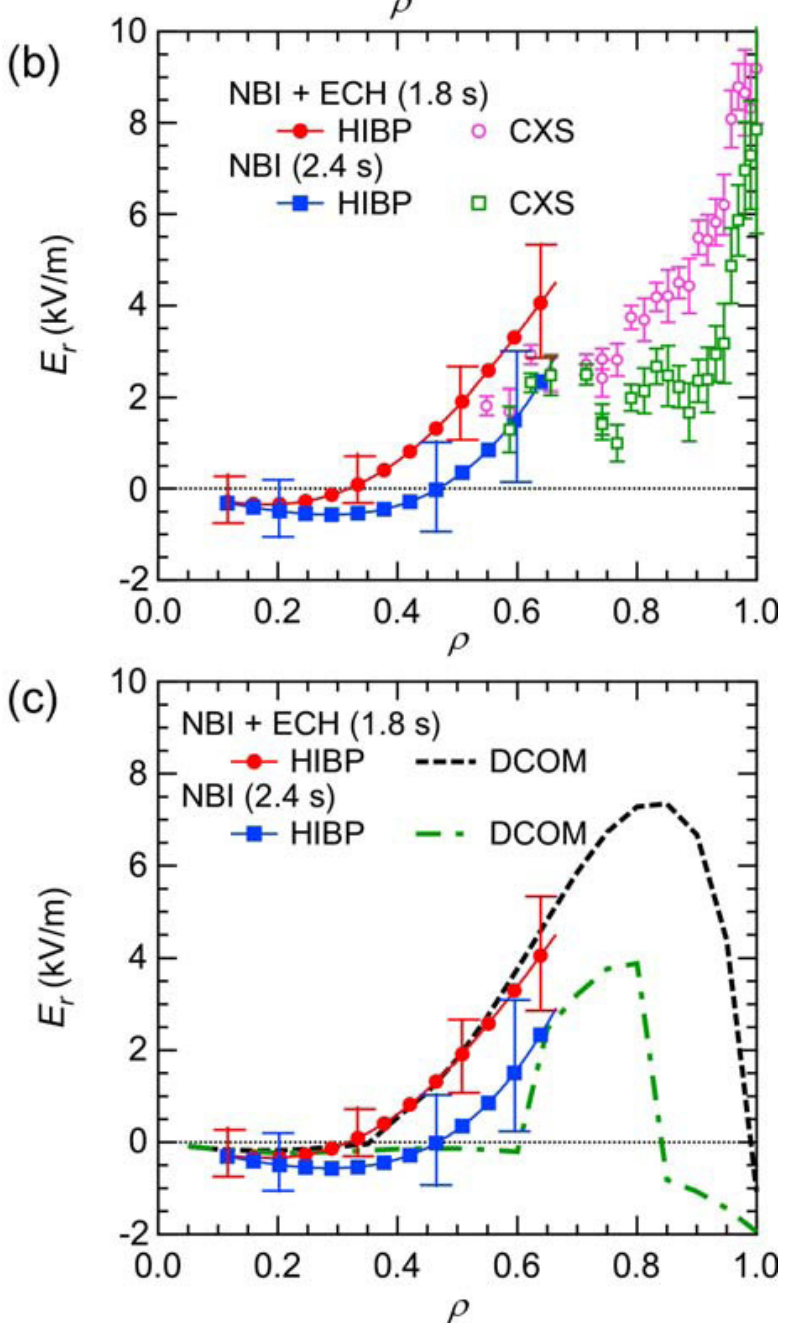

Fig. 8 (a) Potential profiles measured using the HIBP. The horizontal axis is the same as that in Fig. 7 (c). "Gas" is a reference profile measured after the shot. Thus, it indicates the potential of the vacuum vessel of LHD. (b) Comparison of $E_{\mathrm{r}}$ profiles measured using the HIBP and CXS. (c) Comparison of $E_{\mathrm{r}}$ profiles measured using the HIBP and calculated by the DCOM code based on the neoclassical theory. The latter profiles are labeled "DCOM".

from the outside of the observed position contaminates the signal in this phase, $E_{\mathrm{r}}$ measured using the HIBP agrees with that by $\mathrm{CXS}$ at $\rho \sim 0.6$. The results indicate that $E_{\mathrm{r}}$ profiles can be measured successfully across whole radius of the plasma by complimentary use of HIBP and CXS.

In a helical system, $E_{\mathrm{r}}$ can be predicted by the neoclassical theory. Hence, $E_{\mathrm{r}}$ profiles calculated using the Monte-Carlo simulation code DCOM [36] are also compared with those measured using the HIBP in Fig. 8 (c). The calculated $E_{\mathrm{r}}$ agrees with that measured by HIBP. The calculation predicts the transition from the ion root to electron root at $\rho \sim 0.6$ during the NBI phase. $E_{\mathrm{r}}$ measured using the HIBP also changes from weakly negative to positive at about $\rho=0.6$. The results indicate that the DCOM code simulates the $E_{\mathrm{r}}$ profiles measured using the HIBP in the core region, although there is a difference between the experimental and calculation results in the edge region; the reason for this is not yet clear.

\section{Possibility of Fluctuation Measure- ment}

One of the advantages of HIBPs is high temporal resolution measurement. Thus, measurements of potential and density fluctuations have been attempted [37].

Figure 9 shows the temporal behaviors of the line averaged density, heating power, intensity of the secondary beam of the HIBP (which indicates the density fluctuation in plasma), its frequency spectrum, and the potential signal. The secondary beam current is usually detected with a set of four split plates. In this shot, however, only one of the four split plates was connected to an amplifier with high frequency response $(\sim 100 \mathrm{kHz})$. Thus, the potential and density fluctuations cannot be separated in the frequency range $>10 \mathrm{kHz}$. The measurement position is near the plasma center, but the fluctuation of the secondary beam intensity possibly includes fluctuations along the beam trajectory; this is called the path integral effect. According to a simulation, the magnitude of the effect depends on the correlation length of the fluctuation [38]. Thus, we do not discuss the locality of the fluctuation here.

In Fig. 9 (c), coherent fluctuations of the beam intensity appear in the frequency range of 15 to $30 \mathrm{kHz}$. One fluctuation exists from 0.45 to $0.52 \mathrm{~s}$ with a frequency of about $20 \mathrm{kHz}$ and normalized amplitude of $1.8 \%$. The other coherent fluctuation appears at $0.72 \mathrm{~s}$; its frequency increases rapidly from 15 to $25 \mathrm{kHz}$ and then decreases gradually to $21 \mathrm{kHz}$, and its normalized amplitude is 0.6 $\%$. The tendency of the change in the frequency of the latter fluctuation seems to correlate with the behavior of the potential. The correlation may indicate that the frequency change reflects a change in the Doppler shift due to the $E \times B$ flow. The measurement of $E_{\mathrm{r}}$ (potential profile) is necessary to investigate the behavior of the fluctuation.

Thus far, we have detected fluctuations with normalized amplitude of about $0.6 \%$, and the noise level of the power spectrum is about $0.4 \%$. Improvement of the $\mathrm{S} / \mathrm{N}$ ratio is necessary to measure turbulent fluctuation in the core region. After the above-mentioned experiments, we 


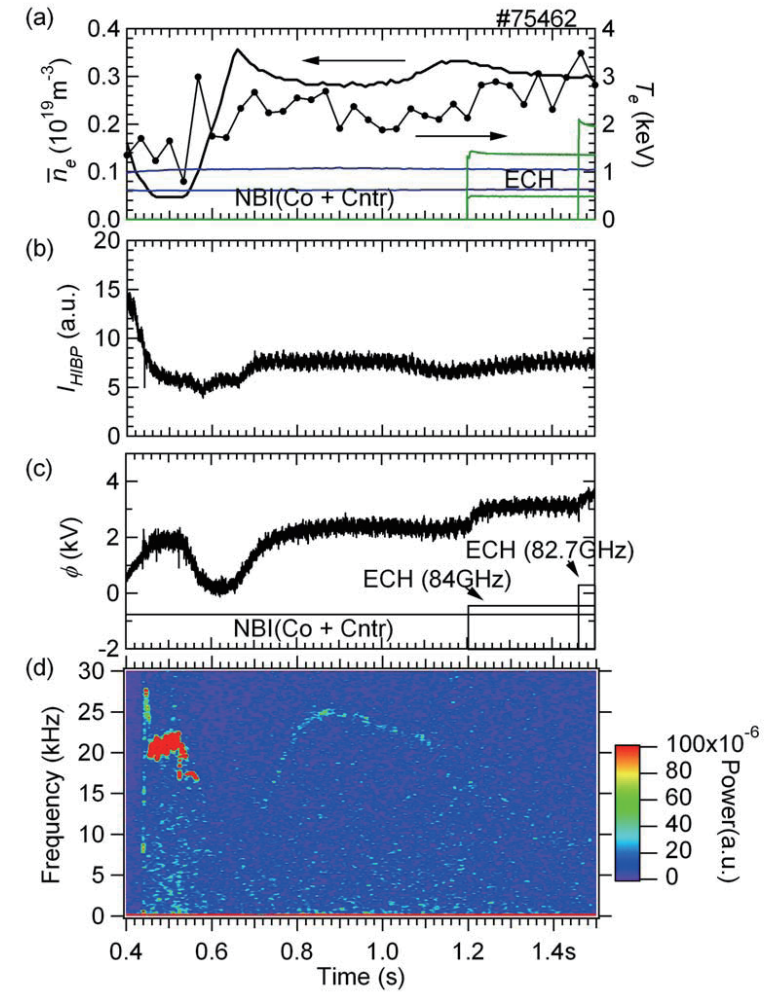

Fig. 9 (a) Temporal evolution of line-averaged electron density and electron temperature in the plasma center. (b) Secondary beam intensity of HIBP. (c) Temporal evolution of the potential signal measured using the HIBP and timing of NBI and ECH. (d) Power spectrum of the fluctuation in the potential signal. Note that the fluctuation with the frequency of $>10 \mathrm{kHz}$ may be contaminated with density fluctuations.

have increased the primary beam current a few times by improving the gas cell in the accelerator and the efficiency of beam transport. The detection efficiency was also increased by $60 \%$ and the noise in the amplifier was reduced. The output of the negative ion source could be increased by increasing the discharge current in the ion source. Therefore, precision of the fluctuation measurement will be improved in the near future.

\section{Summary}

An HIBP using a 3-MV tandem accelerator has been installed in LHD and is calibrated as an integrated system to measure the electrostatic potential in plasmas.

$E_{\mathrm{r}}$ measured directly using the HIBP agrees with that estimated by CXS near the half radius of the plasma. Since the HIBP can access the core region where CXS has difficulty measuring $E_{\mathrm{r}}$ and CXS can access the edge region where HIBP has difficulty measuring $E_{\mathrm{r}}, E_{\mathrm{r}}$ profiles can be measured across the entire radius of the plasma by combining the HIBP and CXS measurements. Therefore, the complementary measurement involving both the HIBP and CXS and high temporal resolution measurement using the HIBP will advance the study of the physics of $E_{\mathrm{r}}$ formation in helical plasmas.

\section{Acknowledgments}

We are grateful to Drs. A. Fujisawa and H. Iguchi of NIFS and Professor K. Ishii of Tsukuba University for useful discussions, and Professors O. Motojima, S. Sudo and K. Itoh for continuous encouragement. We thank Professor T. Watanabe for accurate calculation of the magnetic field in LHD. We thank the LHD technical group for their support.

This work has been supported by MEXT Japan under Grant-in-Aid for Young Scientists (16760674 and 18760640) and by NIFS under NIFS04ULBB505, 507, and 508 .

[1] R.L. Hickok, Rev. Sci. Instrum. 38, 142 (1967).

[2] F.C. Jobes and R.L. Hickok, Nucl. Fusion 10, 195 (1970).

[3] J.C. Hosea et al., Phys Rev. Lett. 30, 839 (1973).

[4] F.M. Bieniosek et al., Rev. Sci. Instrum. 51, 206 (1980).

[5] P.M. Schoch et al., Rev. Sci. Instrum. 57, 1825 (1986).

[6] G.A. Hallock et al., Phys. Rev. Instrum. 56, 1248 (1986).

[7] R.L. Hickok et al., Rev. Sci. Instrum. 59, 1685 (1988).

[8] K. Takasugi et al., Jpn. J. Appl. Phys. 23, 364 (1984).

[9] K. Ishii et al., Rev. Sci. Instrum 60, 3270 (1989).

[10] J.J. Zielinski et al., Rev. Sci. Instrum. 61, 2961 (1990).

[11] A. Fujisawa et al., Rev. Sci. Instrum. 63, 3694 (1992).

[12] H. Iguchi et al., Jpn. J. Appl. Phys. 32, 1829 (1993).

[13] Y. Hamada et al., Plasma Phys. Control. Fusion 36, 1743 (1994).

[14] A.V. Melnikov, Czech. J. Phys. 49, 35 (1999).

[15] T. Ido et al., Rev. Sci. Instrum. 70, 955 (1999).

[16] D.R. Demers et al., Czech. J. Phys. 51, 1065 (2001).

[17] A. Fujisawa et al., Phys. Rev. Lett. 82, 2669 (1999).

[18] H. Maasberg et al., Phys. Plasmas 7, 295 (2000).

[19] T. Shimozuma et al., Plasma Phys. Control. Fusion 45, 1183 (2003).

[20] Y. Takeiri et al., Phys. Plasmas 10, 1788 (2003).

[21] K. Ida et al., Phys. Rev. Lett. 91, 085003 (2003).

[22] T. Estrada et al., Plasma Phys. Control. Fusion 46, 277 (2004).

[23] M. Yokoyama et al., Nucl. Fusion 47, 1213 (2007).

[24] A. Fujisawa, IEEE Trans. Plasma Sci. 22, 395 (1994).

[25] T. Ido et al., Rev. Sci. Instrum. 77, 10F523 (2006).

[26] A. Shimizu et al., Plasma Fusion Res. 2, S1098 (2007).

[27] M. Sasao et al., Rev. Sci. Instrum. 63, 2726 (1992).

[28] A. Taniike et al., IEEE Trans. Plasma Sci. 22, 430 (1994).

[29] M. Nishiura et al., Rev. Sci. Instrum. 77, 03 A537 (2006).

[30] A.Taniike, M. Sasao, J. Fujita, Y. Hamada and M. Wada, Fusion Eng. Des. 34-35, 675 (1997).

[31] T. Ido et al., Plasma Fusion Res. 2, S1100 (2007).

[32] A. Fujisawa et al., Rev. Sci. Instrum. 67, 3099 (1996).

[33] A. Shimizu, Plasma Fusion Res. 2, S1098 (2007).

[34] T.S. Green and G.A. Proca, Rev. Sci. Instrm. 41, 1409 (1970).

[35] Y. Hamada et al., Rev. Sci. Instrm. 68, 2020 (1997).

[36] A. Wakasa et al., J. Plasma Fusion Res. SERIES 4, 408 (2001).

[37] A. Shimizu et al., Proc. 17th international Toki Conference (2007).

[38] H. Nakano et al., Proc. 17th international Toki Conference (2007). 